COMPUTATIONAL METHODS IN APPLIED MATHEMATICS, Vol.9(2009), No.3, pp. 221-225

(C) 2009 Institute of Mathematics of the National Academy of Sciences of Belarus

\title{
POSITIVE DEFINITE HANKEL MATRICES USING CHOLESKY FACTORIZATION
}

\author{
S. AL-HOMIDAN ${ }^{1}$ AND M. ALSHAHRANI ${ }^{1}$
}

\begin{abstract}
Real positive definite Hankel matrices arise in many important applications. They have spectral condition numbers which exponentially increase with their orders. We give a structural algorithm for finding positive definite Hankel matrices using the Cholesky factorization, compute it for orders less than or equal to 30 , and compare our result with earlier results.
\end{abstract}

2000 Mathematics Subject Classification: 65F99, 99C25, 65K30.

Keywords: Cholesky factorization, Hankel matrices, real positive definite matrices.

\section{Introduction}

An $n \times n$ Hankel matrix $H$ is one with constant antidiagonal elements. It has the following structure:

$$
H=\left[\begin{array}{llllll}
h_{1} & h_{2} & h_{3} & \cdots & \cdots & h_{n} \\
h_{2} & h_{3} & h_{4} & \cdots & h_{n} & h_{n+1} \\
\vdots & \cdots & \ddots & \ddots & \ddots & \vdots \\
h_{n} & h_{n+1} & \cdots & \cdots & \cdots & h_{2 n-1}
\end{array}\right]
$$

The general complex-valued elements can be well-conditioned while real positive definite Hankel matrices are known to be very ill-conditioned since their spectral condition numbers increase exponentially with $n$. The condition number of a positive definite Hankel matrix is bounded below by $3 \cdot 2^{(n-6)}$ which is very large even for relatively small orders [9]. Beckermann [6] gave a better bound $\gamma^{n-1} /(16 n)$ with $\gamma \approx 3.210$. Here, we try to force the condition number of the constructed matrix to be as small as possible to reach the latter, or at least the former, experimentally.

Several other authors have developed algorithms for the factorization or inversion of Hankel matrices $[7,8,10]$. In some application areas such as digital signal processing and control theory, it is required to compute the closest, in some sense, positive semidefinite Hankel matrix with no restriction on its rank to a given data covariance matrix computed from a data sequence. The problem of preserving the structure while approximating low rank also arises in many applications (see [1-3]). When using the interior point method in solving this problem, it is important for some algorithms to start from within the cone of positive semidefinite Hankel matrices, i.e., the initial point must be a positive definite Hankel matrix $[4,5]$.

${ }^{1}$ Department of Mathematics and Statistics, King Fahd University of Petroleum and Minerals, Dhahran 31261, Saudi Arabia. E-mail: homidan@kfupm.edu.sa, mshahrani@kfupm.edu.sa 
In this paper, we construct a positive definite Hankel matrix. The Cholesky factorization theorem states that for any $n \times n$ real symmetric positive definite matrix $A$, there exists a unique lower triangular matrix $L$ with positive diagonal entries such that $A=L L^{\top}$. This provides a means to construct a symmetric positive definite matrix by building up a lower triangular $L$ with positive diagonal entries. The matrix $L L^{\top}$ is certainly symmetric positive definite but not Hankel. To impose the Hankel structure, we force the antidiagonal elements of $L L^{\top}$ to be equal. This produces a system of nonlinear equations with infinitely many solutions depending on the diagonal elements of $L$ and the elements of the first diagonal (the diagonal just below the main diagonal). A closed formula for finding the elements of $L$ that makes $L L^{\top}$ positive definite Hankel matrix is introduced in Section 2. In Section 3, an algorithm is proposed and implemented using MatLab. Finally, in Section 4, some numerical comparisons and experiments are presented to show how different choices of starting elements affect the smallest eigenvalue and subsequently the condition number of the resulting Hankel matrix.

\section{Generating Formula}

Let us consider the Cholesky factorization $H=L L^{\top}$ and write down the product. The resulting matrix $L L^{\top}$ is as follows:

$$
\left[\begin{array}{lllll}
l_{11}^{2} & l_{11} l_{21} & l_{11} l_{31} & \cdots & l_{11} l_{n 1} \\
l_{11} l_{21} & l_{21}^{2}+l_{22}^{2} & l_{21} l_{31}+l_{22} l_{32} & \cdots & l_{21} l_{n 1}+l_{22} l_{n 2} \\
l_{11} l_{31} & l_{21} l_{31}+l_{22} l_{32} & l_{31}^{2}+l_{32}^{2}+l_{33}^{2} & \cdots & l_{31} l_{n 1}+l_{32} l_{n 2}+l_{33} l_{n 3} \\
\vdots & \vdots & \vdots & \ddots & \vdots \\
l_{11} l_{n 1} & l_{21} l_{n 1}+l_{22} l_{n 2} & l_{31} l_{n 1}+l_{32} l_{n 2}+l_{33} l_{n 3} & \cdots & l_{n 1}^{2}+l_{n 2}^{2}+\cdots+l_{n n}^{2}
\end{array}\right] .
$$

This is a dense matrix. Now, we show how to find the elements of $L$ which make $L L^{\top}$ a positive definite Hankel matrix.

- First, we assign values to all the elements $l_{i i}$ and $l_{i \text { i-1 }}$.

- Each computed element $l_{i j}$ has the form $(\alpha-\beta) / l_{j j}$, where

- $\alpha$ is composed of summing products of pairs of elements $l_{h s} l_{k s}$, where $h=[n / 2]$ $([x]$ is an integer smaller than or equal to $x)$ and $k=n-h$ and the index $s$ runs from 1 to $h$. So $\alpha$ is related to $i+j$;

$-\beta$ is also a sum of products of pairs $l_{j r} l_{i r}$, where $r$ runs from 1 to $j-1$. So $\beta$ is related to $j$ only.

- The last two elements $l_{n n-1}$ and $l_{n n}$ are free in the sense that no other elements depend on them.

In the light of the above, the following two steps construct $L$ such that $L L^{\top}$ is a positive definite Hankel matrix

- Assign values to $l_{i i}$ and $l_{i \text { i-1 }}$. 
- Compute the other elements using the formula

$$
l_{i j}=\frac{1}{l_{j j}}\left(\sum_{s=1}^{q} l_{q, s} l_{r s}-\sum_{t=1}^{j-1} l_{i t} l_{j t}\right),
$$

where $j \leqslant i-2$ and

$$
q=\left\{\begin{array}{ll}
\frac{i+j}{2}, & \text { if } i+j \text { even, } \\
\frac{i+j-1}{2}, & \text { if } i+j \text { odd }
\end{array} \quad r= \begin{cases}q, & \text { if } i+j \text { even } \\
q+1, & \text { if } i+j \text { odd }\end{cases}\right.
$$

\section{Algorithm}

The following algorithm presents formula (2.1) in a more programmable way. It produces all the elements of $L$ such that $L L^{\top}$ is a positive definite Hankel matrix.

Algotithm 3.1 (Constructing Positive Definite Hankel Matrix).

\section{begin}

for $i:=1$ to $n$

populate $l_{i i}$ and $l_{i-1}$

for $i:=3$ to $n$

$$
\begin{gathered}
\text { for } j:=1 \text { to } i-2 \\
\text { set } q:=[(i+j) / 2] \\
\text { if } n \text { is even } \\
\text { set } r:=q \\
\text { else } \\
\text { set } r:=q+1 \\
\text { set } \alpha:=0 \\
\text { set } \beta:=0 \\
\text { for } s:=1 \text { to } q \\
\alpha=\alpha+l_{q s} l_{r s} \\
\text { for } t:=1 \text { to } j-1 \\
\beta=\beta+l_{i t} l_{j t} \\
\text { set } l_{i j}:=(\alpha-\beta) / l_{j j} .
\end{gathered}
$$

\section{Numerical Experiments}

The first experiment is to find the highest order of $H$ we can produce. Varah [10] managed to get up to $n=16$, whereas our program managed to construct a positive definite Hankel matrix $H$ of order $n=30$ with the smallest eigenvalue $e=5.18 \times 10^{-15}$.

For the remaining experiments, we fix the order to be 20 and we choose different values for $l_{i i}$ and $l_{i i-1}$ to improve the condition number. The lower bound mentioned in [9] for $n=20$ is 49152 while the bound in [6] is 3624.29. The goal of our experiment is to push down the condition number towards these bounds.

The Matlab implementation of Algorithm 3.1 takes a $1 \times 5$ vector $a$, where $a_{1}$ is assigned to $l_{11} ; a_{2}$ is assigned to $l_{i i}$, for $i=2, \ldots, n-1 ; a_{3}$ is assigned to $l_{i-1}$, for $i=2, \ldots, n-1$; $a_{4}$ is assigned to $l_{n n-1}$; and $a_{5}$ is assigned to $l_{n n}$. 
First, we change $a_{1}$ only and hold the other elements constant (with 1 as the initial value). Table 4.1 shows that the condition number $\kappa(H)$ improves when $a_{1}$ is changed from 1 to 10 , but then gets worse when we go higher.

Table 4.1. Changing $\boldsymbol{a}_{1}$

\begin{tabular}{|c|c|c|}
\hline$a_{1}$ & $e$ & $\kappa(H)$ \\
\hline 1 & $6.27 \times 10^{-8}$ & $7.55 \times 10^{17}$ \\
10 & $1.65 \times 10^{-7}$ & $4.58 \times 10^{16}$ \\
100 & $1.61 \times 10^{-7}$ & $7.17 \times 10^{16}$ \\
1000 & $1.60 \times 10^{-7}$ & $7.56 \times 10^{16}$ \\
\hline
\end{tabular}

Now, we change $a_{1}$ and $a_{5}$ together and hold the other elements constant. Clearly, from Table 4.2 , the best choice is $a_{1}=a_{5}=10$.

Table 4.2. Changing $\boldsymbol{a}_{\mathbf{1}}$ and $\boldsymbol{a}_{\mathbf{5}}$

\begin{tabular}{|c|c|c|c|}
\hline$a_{1}$ & $a_{5}$ & $e$ & $\kappa(H)$ \\
\hline 10 & 10 & $4.20 \times 10^{-7}$ & $1.80 \times 10^{16}$ \\
100 & 100 & $4.09 \times 10^{-7}$ & $2.82 \times 10^{16}$ \\
1000 & 1000 & $4.07 \times 10^{-7}$ & $2.97 \times 10^{16}$ \\
\hline
\end{tabular}

After some testing, the following combination in Table 4.3 seems to give better results with the condition number reduced to $9.15 \times 10^{14}$.

Ta ble 4.3. Changing $\boldsymbol{a}_{1}, \ldots, \boldsymbol{a}_{5}$

\begin{tabular}{|c|c|c|}
\hline$a$ & $e$ & $\kappa(H)$ \\
\hline$\left[\begin{array}{cll}100000 & 5000-9010100000\end{array}\right]$ & 14.14 & $9.15 \times 10^{14}$ \\
{$[10000005000-90101000000]$} & 14.18 & $9.11 \times 10^{14}$ \\
{$[1000000050000-901000010000000]$} & 1417.8 & $9.09 \times 10^{14}$ \\
\hline
\end{tabular}

After testing different values of $l_{i i}$ and $l_{i-1}$ with different $n$, we find that if we normalize all values of the matrix $L$ so that all the elements of $L$ and $H$ are less than or equal to one, then the best condition number can be reached by selecting the elements of $l_{i-1}$ equal to either one or zero. The elements $l_{i i}$ are selected as follows:

Start with $l_{11}=0.9$, the rest of the diagonal elements of $L$ is generated by multiplying the pervious diagonal element by a factor, i.e., $l_{i i}=f * l_{i-1}{ }_{i-1}$, where $f$ is a factor. We choose this factor to be $f=0.5$ for $i=2, \ldots, n / 2$. Then the factor is increased as $i$ increases up to $n$ in the following way: from $n / 2$ till $2 n / 3$, the factor $f$ is increased gradually from 0.5 to 1.0 . From $2 n / 3$ till $n$, the factor $f$ is increased exponentially so that $l_{n n}=150 l_{k k}$, where $k=2 n / 3$.

We implement this strategy for the above case $(n=20)$ and find a remarkable improvement to the condition number as follows:

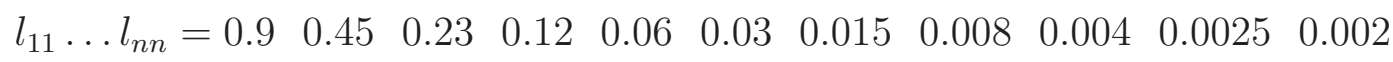

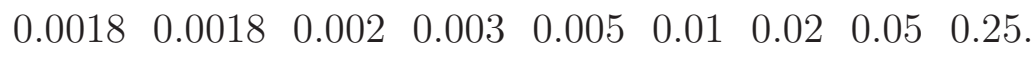

The condition number we find is $7.2776 \times 10^{8}$ which is much better while the smallest eigenvalue is $2.2945 \times 10^{-9}$. It is worth mentioning that the condition number we find for $n=3, \ldots, 16$ is very close to the one found by $[10]$. 


\section{Conclusions}

In this paper, we managed to construct a positive definite Hankel matrix with a smaller eigenvalue greater than zero, and we improved the condition number by a factor of 100 . However, the produced matrices are extremely ill-conditioned and more work needs to be done to deduce a pattern that improves the results. Also, a higher dimension of $H$ is needed. One way to do it is to decrease the tolerance by giving the program more accuracy, but this will increase the dimension by one or two since it is clear that the condition number increases exponentially while the smallest eigenvalue also decreases exponentially.

Acknowledgements. The authors are grateful to King Fahd University of Petroleum and Minerals, Dharhan, Saudi Arabia for providing excellent research facilities. This research was supported by the Fast Track Research Project no. FT080013.

\section{References}

1. Suliman Al-Homidan, Combined methods for approximating Hankel matrix, WSEAS Trans. Syst., 1 (2002), no. 1, pp. 35-41.

2. Suliman Al-Homidan, Hybrid methods for approximating Hankel matrix, Numer. Algorithms, 32 (2003), no. 1, pp. 57-66.

3. Suliman Al-Homidan, Structured methods for solving Hankel matrix approximation problems, Pac. J. Optim., 1 (2005), no. 3, pp. 599-609.

4. Suliman Al-Homidan, Solving Hankel matrix approximation problem using semidefinite programming, Journal of Computational and Applied Mathematics, 202 (2007), no. 2, pp. 304-314.

5. Mohammed M. Alshahrani and Suliman S. Al-Homidan, Mixed semidefinite and second-order cone optimization approach for the Hankel matrix approximation problem, Nonlinear Dyn. Syst. Theory, 6 (2006), no. 3, pp. 211-224.

6. B. Beckermann, The condition number of real Vandermonde, Krylov and positive definite Hankel matrices, Numer. Math., 85 (2000), no. 4, pp. 553-577.

7. D. L. Boley, F. T. Luk, and D. Vandevoorde, Vandermonde factorization of a Hankel matrix, In Scientific computing (Hong Kong, 1997), Springer, Singapore, 1997, pp. 27-39.

8. V. Olshevsky and M. Stewart, Stable factorization for Hankel and Hankel-like matrices, Numer. Linear Algebra Appl., 8 (2001), no. 6-7, pp. 401-434.

9. E. E. Tyrtyshnikov, How bad are Hankel matrices? Numer. Math., 67 (1994), no. 2, pp. 261-269.

10. J. M. Varah, Positive definite Hankel matrices of minimal condition, Linear Algebra Appl., 368 (2003), pp. 303-314. 